\title{
Cross-Shelf Transport Through the Interaction among a Coastal Jet, a Topographic Wave, and Tides
}

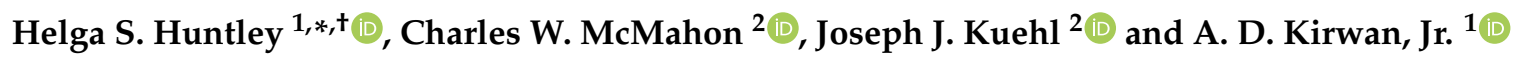 \\ 1 School of Marine Science \& Policy, University of Delaware, Newark, DE 19711, USA; adk@udel.edu \\ 2 Department of Mechanical Engineering, University of Delaware, Newark, DE 19711, USA; \\ mcmahon@udel.edu (C.W.M.); jkuehl@udel.edu (J.J.K.) \\ * Correspondence: huntleyh@rowan.edu or helgah@udel.edu \\ + Current address: Department of Mathematics, Rowan University, Glassboro, NJ 08028, USA.
}

Received: 14 July 2020 ; Accepted: 12 October 2020; Published: 16 October 2020

\begin{abstract}
Shelf break flows are often characterized by along-isobath jets with cross-shelf currents associated with tides and waves guided by variable topography. Here, we address the question: Can a superposition of such flows produce significant aperiodic cross-shelf transport? To answer this question, we use a barotropic analytic model for the jet based on a similarity solution of the shallow water equations over variable topography, a wave disturbance determined by the topography, and a diurnal tidal disturbance. We use standard Lagrangian methods to assess the cross-shelf transport, presenting the results, however, in a Eulerian frame, so as to be amenable to oceanographic observations. The relative roles of the different flow components in cross-shelf transport are assessed through an extensive parameter study. We find that a superposition of all three flow components can indeed produce consequential background aperiodic transport. An application of the model using recent observations from the Texas Shelf demonstrates that a combination of these background mechanisms can produce significant transport under realistic conditions.
\end{abstract}

Keywords: cross-shelf transport; topographic Rossby waves; finite time Lyapunov exponents; Texas-Louisiana Shelf

\section{Introduction}

The outer shelf and shelf break constitute some of the most important and challenging regions of the world oceans. As discussed by Brink [1], the importance arises from the fact that this is where significant amounts of nutrients, biological matter, pollutants, and other material are exchanged between coastal regions and the deep ocean. Concurrently, observing and predicting significant exchange events in this region is challenging. As documented in some detail 25 years ago by Huthnance [2] and more recently by Brink [1], the difficulties arise from the plethora of physical processes operating in this zone and their disparate time and space scales.

Generally, the flow along shelf breaks is composed of relatively energetic along-isobath currents with cross-shelf flows associated with tides and turbulent processes. Moreover, atmospheric processes and the interactions of this flow with deep-ocean eddies may produce intense, yet brief cross-shelf exchange and significant upwelling and vertical transport [3]. These mechanisms combine to produce significant inhomogeneity in the spatial structure of fields, such as temperature, salinity, and other tracers, and of the currents themselves. The consequent range of time and space scales involved is sufficiently large to pose challenges for all but the most comprehensive field programs, as well as for basin-scale general circulation models to resolve.

Prior studies of the exchange across shelf regions, such as reported in [4,5], focused on property gradients and mixing coefficients based on climatological data, hydrographic cruises, and moorings. 
These studies were useful for establishing seasonal to climatological trends in specific geographic regions. However, as demonstrated by the application to transport of oil into near-shore waters during the Deepwater Horizon oil spill [6], such data do not adequately account for the transport across the shelf even in relatively quiescent periods. This suggests that there may be important but heretofore overlooked background cross-shelf transport processes.

To fill this gap, we test the hypothesis that aperiodic cross-shelf transport can arise from a combination of simple periodic processes that frequently form the background shelf circulation. For present purposes, it is sufficient to use a model that considers just three processes, specifically the interaction of a coastal current modulated by a topographic wave disturbance and tides. Each process by itself can only lead to periodic fluid exchange. Even a combination of any two of these is insufficient; but as we shall demonstrate, together, these three processes have the potential to lead to significant aperiodic cross-shelf water exchange.

As transport is essentially a Lagrangian process, we employ appropriate Lagrangian methods to elucidate particle behavior in such a flow. Since the early work of Bower [7], Samelson [8], and Rogerson et al. [9] on fluid exchange across meandering jets, the application of these methods has produced significant progress in understanding transport in geophysical flows. See also Wiggins [10], Haller [11], Samelson and Wiggins [12], Budyansky et al. [13], and Pratt et al. [14] for more recent results and references. Here, we extend this approach to jet flow over a sloping bottom. However, we choose to present the results relative to a Eulerian reference to make our results more amenable to oceanographic observations.

The balance of this report is organized as follows. The next section reviews the model and analysis methods. A base case with typical parameter values is analyzed in detail and basic transport properties described in Section 3. This is followed by an extensive exploration of the model parameter space in Section 4. In Section 5, we apply the model to recent data from the Texas-Louisiana Shelf. This region is chosen because it is generally typical of shelf and upper slope regions in the world and because of the availability of recent hydrographic data. Our report concludes in Section 6 with a summary and discussion of some broader implications for cross-shelf transport.

\section{Model and Methods}

In order to analyze the transport associated with tidal oscillations in the presence of a classic coastal jet with a topographic Rossby wave disturbance, we turn to the theoretical framework developed by Kuehl [15]. This setup leads to an analytic solution for the transport function and hence the velocity field. Trajectories are computed numerically, using a standard explicit fourth order Runge-Kutta scheme. Details of the model are provided in Section 2.1, followed by the methodology to identify Lagrangian structures in Section 2.2 and for transport quantification in Section 2.3.

\subsection{The Model}

The flow of interest consists of the following components: (1) a coastal jet in the form of an along-slope mean flow with peak velocity located offshore; (2) a traveling wave, such as a topographic Rossy wave, with a multi-day period; and (3) a higher frequency tidal oscillation. Here, we will consider the simplest case of a barotropic flow along slope topography with Ekman dissipation [15]. Note that extensions to the non-linear case [16] and to stratified flow [17] are relatively straightforward, but beyond the scope of the present study. 
We adopt a right-handed coordinate system with $x$ in the along-shore direction and $y$ in the cross-shore direction, where $y=0$ at the center of the coastal jet. The coastal jet is typically directed in the negative $x$-direction, with shallower waters for higher values of $y$. See Figure 1 . In the region of interest, the bathymetry is taken to be a simple constant slope of the form:

$$
h=h_{0}-S y,
$$

for constants $h_{0}$ and $S$. The three flow components are assumed to be linearly additive and are modeled individually. The linear superposition assumption is justified when the background flow is small. Within the jet core, the relative vorticity gradients may modify the topographic wave structure, but on the scale of the shelf, topographic effects dominate.

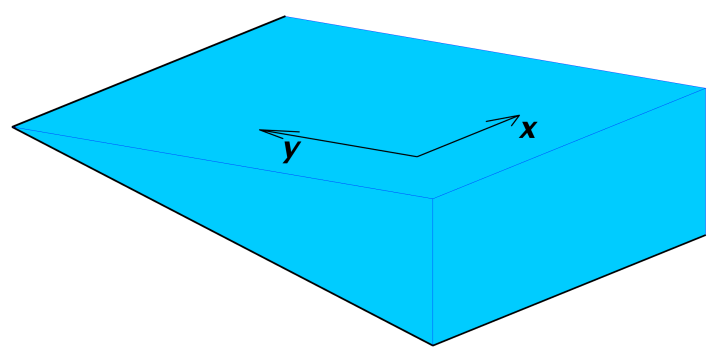

Figure 1. Model coordinate system.

The mean flow is assumed to be steady and linear, with Ekman dissipation in a bottom boundary layer as the dominant viscous term and the cross-stream shear dominating the relative vorticity. Under these conditions, the vorticity-streamfunction formulation of the shallow water equations $[18,19]$ can be solved for the volume-transport streamfunction. The solution takes on the form of an error function:

$$
\Psi_{j e t}=A \operatorname{erf}(\lambda y) .
$$

$A$ determines the strength of the coastal jet, while $\lambda$ parameterizes its width. In general, $\lambda$ varies with $x$, so that the jet widens downstream. In an effort to isolate the lowest order dynamics, we approximate $\lambda$ as a constant, which corresponds to the limit of vanishing dissipation.

The traveling wave can generically be modeled by $\Psi_{\text {wave }}=B \exp \left(i k x+i l y-i \omega_{w} t\right)$. For typical topographic waves, which follow isobaths, $l=0$. To fix the analysis window, a frame of reference is chosen that moves with the wave at phase speed $c_{p}=\omega_{w} / k$. With both of these modifications, the associated volume-transport streamfunction simplifies to:

$$
\Psi_{\text {wave }}=B \cos (k x)+\int_{0}^{y} c_{p} h d y .
$$

$B$ sets the amplitude of the wave disturbance, and $k$ is the wavenumber. For a topographic Rossby wave, the phase speed $c_{p}$ and wavenumber $k$ do not vary independently, because of the dispersion relation (e.g., [20]):

$$
\omega_{w}=-\frac{\beta R^{2} k}{1+R^{2} k^{2}}
$$


Here, $\beta=S f / h_{0}$ is the topographic beta parameter, and $R=\sqrt{g h_{0}} / f$ is the Rossby number, where $g$ is the gravitational constant and $f$ is the Coriolis parameter. For typical values explored here, $R$ and $k$ are sufficiently large that this dispersion relation can be well approximated by:

$$
\omega_{w} \approx-\frac{\beta R^{2} k}{R^{2} k^{2}}=-\frac{\beta}{k} .
$$

Substituting this expression and the definition for $\beta$ into the equation for $c_{p}$ leads to the relationship:

$$
c_{p}=-\frac{S f}{h_{0} k^{2}} .
$$

Other dispersion relations can be implemented to model different topographically controlled waves, such as continental shelf waves.

Finally, the high-frequency tidal oscillations are represented as a generic periodic time dependence in the cross-shelf direction only:

$$
\Psi_{\text {tide }}=C \sin (2 \pi \omega t) x \text {. }
$$

$C$ and $\omega$ indicate the tidal amplitude and frequency, respectively.

Putting the pieces together yields the following volume-transport streamfunction:

$$
\Psi=A \operatorname{erf}(\lambda y)+B \cos (k x)+\int_{0}^{y} c_{p} h d y+C \sin (2 \pi \omega t) x .
$$

The corresponding velocity field takes the form:

$$
\begin{aligned}
& u(x, y, t)=-\frac{1}{h} \frac{\partial \Psi}{\partial y}=-\frac{2}{\sqrt{\pi}} \frac{\lambda A}{h} e^{-\lambda^{2} y^{2}}-c_{p}=-\frac{2}{\sqrt{\pi}} \frac{\lambda A}{h} e^{-\lambda^{2} y^{2}}+\frac{S f}{h_{0} k^{2}}, \\
& v(x, y, t)=\frac{1}{h} \frac{\partial \Psi}{\partial x}=-\frac{k B}{h} \sin (k x)+\frac{C}{h} \sin (2 \pi \omega t) .
\end{aligned}
$$

Thus, the along-shore flow, $u$, consists of the background jet component, plus the effect of the moving frame of reference. The cross-shore flow, $v$, reflects the spatial oscillations of the topographic wave and the temporal oscillations of the tide. It is assumed that the model domain is sufficiently far from the coastal boundary, so that boundary effects do not impact the analysis.

The model is controlled by a total of ten parameters, $A, \lambda, B, k, C, \omega, h_{0}, S, f$, and the time interval $\tau$ over which the volume transport is computed. Not all of these parameters have direct physical interpretations. Therefore, we replace them with ones that do. These are summarized, with their definitions in terms of the basic ten parameters, their physical meaning, units, and base case values, in Table 1. With these derived parameters, the fundamental model equations become:

$$
\begin{aligned}
& u(x, y, t)=-\frac{h_{0}}{h} U e^{-16 y^{2} / L^{2}}-\frac{2 \pi}{T k^{\prime}}, \\
& v(x, y, t)=-\frac{h_{0}}{h} V \sin (k x)+\frac{h_{0}}{h} V_{\text {tide }} \sin (2 \pi \omega t),
\end{aligned}
$$

where

$$
k=-\frac{S f T}{2 \pi h_{0}}
$$

and

$$
f=14.5842 \times 10^{-5} \sin (\phi) .
$$


Table 1. Model parameters, definitions, physical meaning, base case values, and units.

\begin{tabular}{|c|c|c|c|c|}
\hline$\phi$ & $\arcsin \left(\frac{f}{14.5842 \times 10^{-5}}\right)$ & latitude & 43 & (degrees) \\
\hline $\begin{array}{l}h_{0} \\
S\end{array}$ & & water depth at the peak of the coastal jet & $\begin{array}{r}100 \\
0.01\end{array}$ & $(\mathrm{~m})$ \\
\hline$U$ & $\frac{2 \lambda A}{\sqrt{\pi} h_{0}}$ & strength of the coastal jet & 0.05 & $(\mathrm{~m} / \mathrm{s})$ \\
\hline$L$ & $\frac{4}{\lambda}$ & width of the coastal jet & 5000 & (m) \\
\hline$V$ & $\frac{k B}{h_{0}}$ & amplitude of the topographic wave & 0.05 & $(\mathrm{~m} / \mathrm{s})$ \\
\hline$T$ & $\frac{2 \pi}{\left|c_{p} k\right|}$ & topographic wave period & 3 & (days) \\
\hline $\begin{array}{l}V_{\text {tide }} \\
\omega\end{array}$ & $\frac{C}{h_{0}}$ & $\begin{array}{l}\text { amplitude of the tidal oscillation } \\
\text { tidal frequency }\end{array}$ & $\begin{array}{r}0.02 \\
1\end{array}$ & $\begin{array}{c}(\mathrm{m} / \mathrm{s}) \\
\left(\mathrm{day}^{-1}\right)\end{array}$ \\
\hline$\tau$ & & analysis time interval & 3 & (days) \\
\hline
\end{tabular}

\subsection{Lagrangian Structures: Finite-Time Lyapunov Exponents}

One tool to map out the transport properties of a complex flow is the field of finite-time Lyapunov exponents (FTLEs) [21]. FTLEs are a common method for approximating Lagrangian coherent structures (LCS), i.e., manifolds that organize the domain into regions of different flow properties. For detailed discussions of LCS, the reader is referred to the comprehensive synopses by Wiggins [10] and Haller [11] and the references cited therein.

The FTLE is defined as follows: Consider the flow map $\phi$, mapping initial position $x_{0}$ to a final position $\boldsymbol{x}_{f}$ at time T. The Cauchy-Green deformation tensor is defined as $G=\frac{\partial \phi}{\partial x_{0}}\left(\frac{\partial \phi}{\partial x_{0}}\right)^{\prime}$, where $(\cdot)^{\prime}$ denotes the matrix transpose. If the largest eigenvalue of $G$ is $\mu$, then the FTLE is defined as $\lambda=\log (\sqrt{\mu}) / T$. FTLE can be computed in forward and backward time. Ridges in the forward FTLE field (shown in red here) are frequently associated with stable manifolds, while those in the backward FTLE field (shown in blue) generally mimic unstable manifolds (this is not always true: flows undergoing rapid transition can experience transport across FTLE ridges [22]). Intersections of stable and unstable manifolds demarcate hyperbolic regions in the flow. Material approaches such an intersection along the stable manifold and leaves along the unstable manifold.

While the velocity field for the model is analytic, the trajectories underlying our analysis are computed numerically, using a fourth order Runge-Kutta scheme. Similarly, the elements of the strain tensor $F=\frac{\partial \phi}{\partial x_{0}}$ are computed by integrating along trajectories:

$$
F_{i j}=\frac{\partial x_{i}}{\partial x_{0 j}}=\delta_{i j}+\int_{0}^{t} \frac{\partial u_{i}}{\partial x_{0 j}} d \tau=\delta_{i j}+\int_{0}^{t}\left(\sum_{k=1}^{2} \frac{\partial u_{i}}{\partial x_{k}} F_{k j}\right) d \tau,
$$

following Huntley et al. [23]. 


\subsection{Transport Quantification}

For quantifying transport, we compute the area in the horizontal plane of the water masses in question and multiply by the depth. For this purpose, the depth is approximated as stepwise constant over segments $d y=10 \mathrm{~m}$.

A quantitative theoretical tool for Lagrangian transport analyses is lobe dynamics [12]. The basic idea is that a lobe, defined by two consecutive intersection points of the same two manifolds and the manifold segments in between, moves from outside to inside the area of interest. This kind of calculation works best in idealized, e.g., [9,24], or slowly evolving flows, e.g., [25,26], where the manifolds are sharply defined. However, in the present case, this methodology is complicated in part of the parameter space by the hyperbolic regions in the FTLE field being relatively weak and not stationary (see Section 3 and Figure 3), while for different parameter choices, multiple ridges appear in close proximity (not shown), making the problem prone to numerical errors. Significant variations in the Lagrangian structures across the parameter space add further challenges. The best choice for how to define the boundary segments from a Lagrangian analysis for the areas of interest is therefore not clear. Moreover, the primary question we seek to address is the shoreward transport across the jet. Therefore, we chose not to pursue lobe dynamics.

There are two natural options for defining the boundary for the cross-shelf transport. One is the $y=0$ plane, where the center of the unperturbed coastal jet is situated. This is equivalent to transport across the line of constant depth $h=100 \mathrm{~m}$. Due to its connection to a topographic line, this definition of "cross-shelf" transport is easy to apply to observations. The other option is the plane defined by $\Psi=0$ that crosses $y=0$ at $t=0$. This plane moves with the flow, but returns with the tidal period to its initial position. Its main advantage is that it is more directly related to the flow structures. That is, in the $V_{\text {tide }}=0$ case, there is no transport across $\Psi=0$, while there is transient transport across $y=0$. Thus, in this manuscript, we chose to quantify cross-topographic transport relative to $\Psi=0$.

Concretely, the transport calculation is performed by parameterizing the curve $\Psi(t=0)=0$ with 1001 evenly spaced particles over two wave periods that are advected using the analytic velocity field and a fourth order fixed-step Runge-Kutta scheme. As distances between adjacent particles exceed $100 \mathrm{~m}$, new particles are added along a linear interpolation between these particles. The area is then computed between the initial and final realizations of the curve. The transport magnitude is reported as the volume exchanged from onshore to offshore, which is equivalent to the volume exchanged in the other direction.

\section{Base Case}

\subsection{The Flow Field and Its Lagrangian Structures}

For an initial exploration, we use parameter values that are realistic but not tuned to a specific coast. The values are listed in Table 1 . The coastal jet has its peak $10 \mathrm{~km}$ offshore and a width of approximately $5 \mathrm{~km}$. The jet is modified by a strong Rossby wave with a wavelength of approximately $23 \mathrm{~km}$. The tidal frequency is one day. Because of the tide, the velocity field is time dependent even in the moving reference frame we adopted. A snap-shot for $t=0$ is shown in Figure 2a with the corresponding volume-transport streamfunction in Figure $2 b$. 


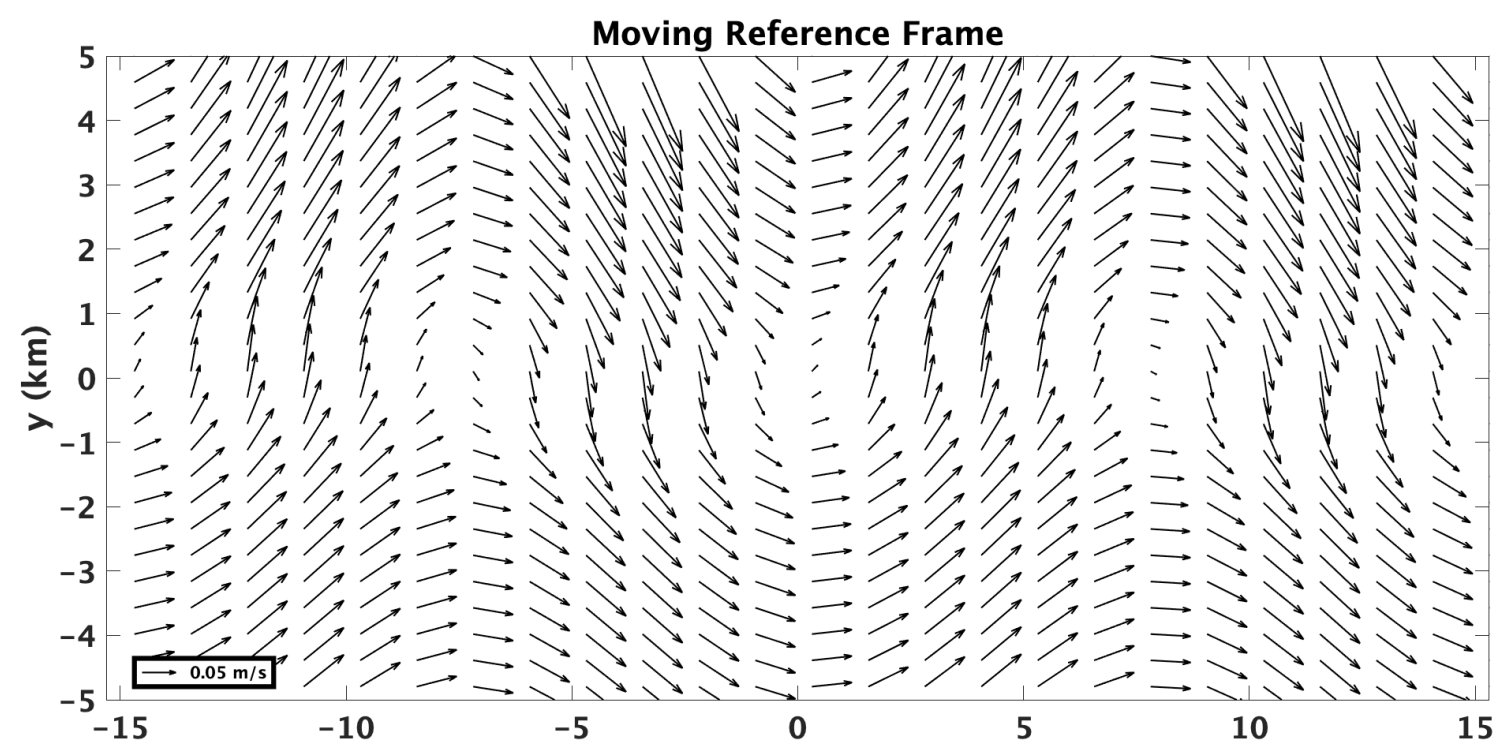

(a)

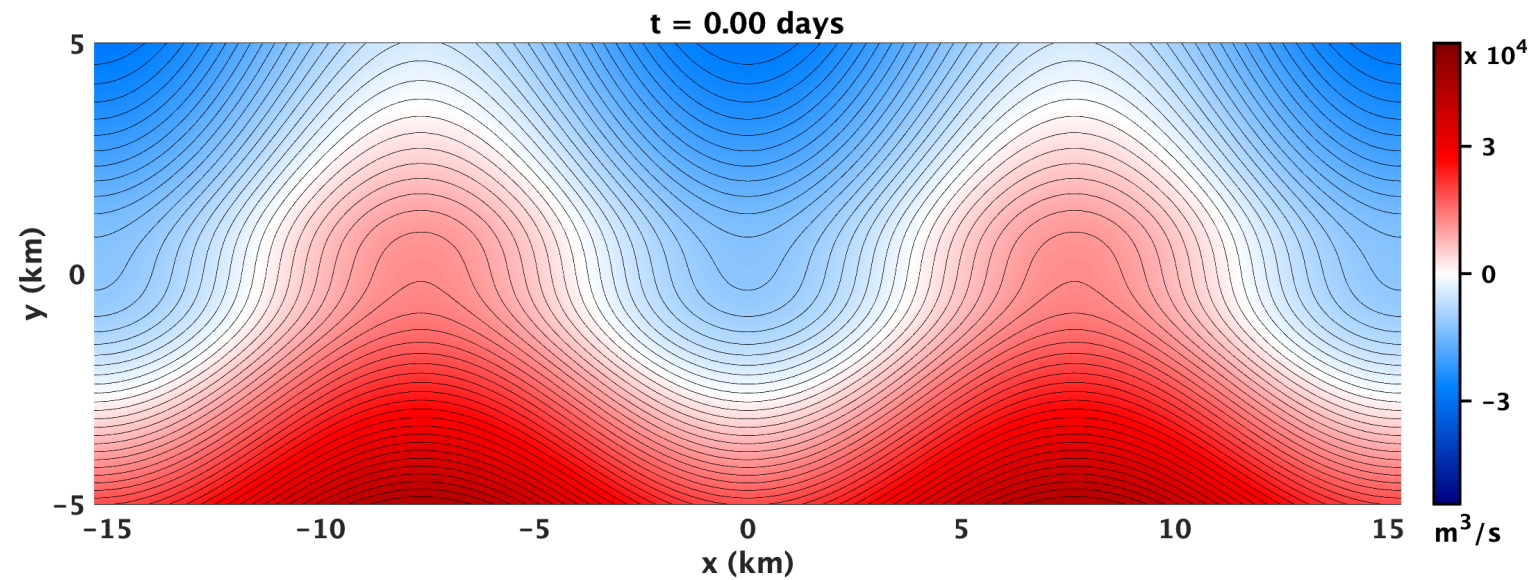

(b)

Figure 2. (a) Model velocity field and (b) volume-transport streamfunction $\Psi$ at $t=0$ for the base case parameters in Table 1.

Even though the three components of the flow field are individually simple, they combine into a complex flow. This is reflected in the evolution of the LCS as visualized by ridges in the FTLE fields. Four snapshots of the FTLE field for our base case are displayed in Figure 3. The FTLEs are characterized at $t=0$ in each interval of one wavelength by one hyperbolic region near $y=-4.4 \mathrm{~km}$ (e.g., at $x=0 \mathrm{~km}$ ), with strong ridges emanating diagonally toward the shore, connecting it to another weakly hyperbolic region near $y=0.08 \mathrm{~km}$ (e.g. at $x= \pm 7.6 \mathrm{~km}$ ). The structure oscillates slightly shoreward and back over a tidal cycle (the other ridges visible in the plots are impacted by edge effects and not necessarily meaningful). Initially, at time $t=0$ (Figure 3a), the ridges are closely associated with the $\Psi=15,000 \mathrm{~m}^{3} / \mathrm{s}$ contour. Over time, however, the Eulerian ( $\Psi$ ) field tilts significantly away from the Lagrangian (FTLE) field (Figure $3 b-d$ ), before coinciding again after one full tidal period. This separation of Eulerian and Lagrangian flow structures is an indication of potential transport across the Eulerian benchmark given by the $\Psi$-isoline. 


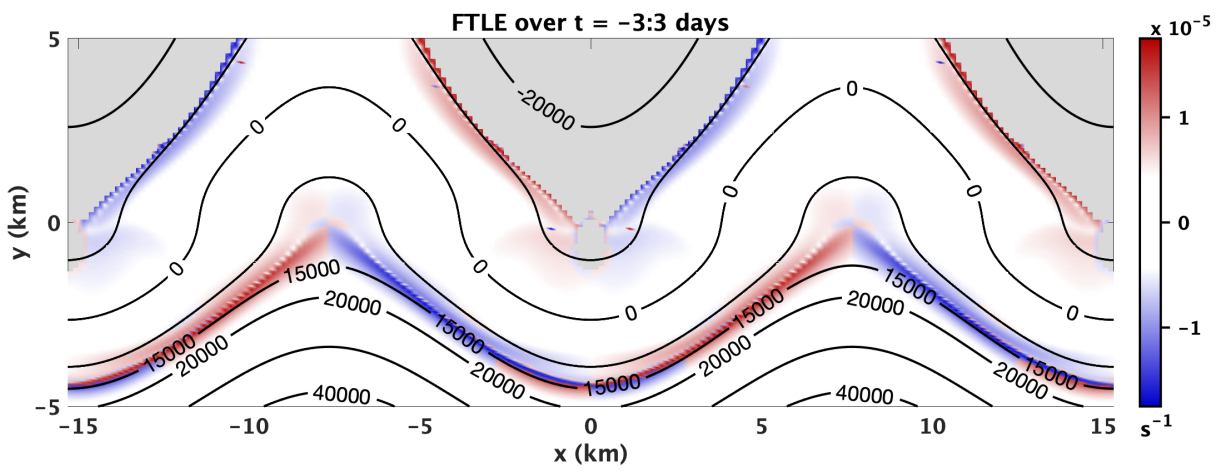

(a)

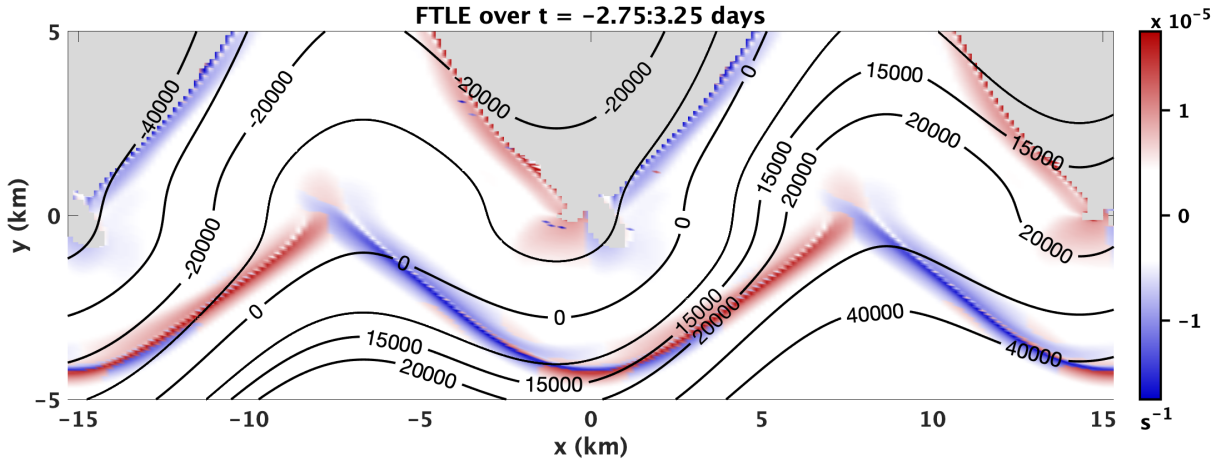

(b)

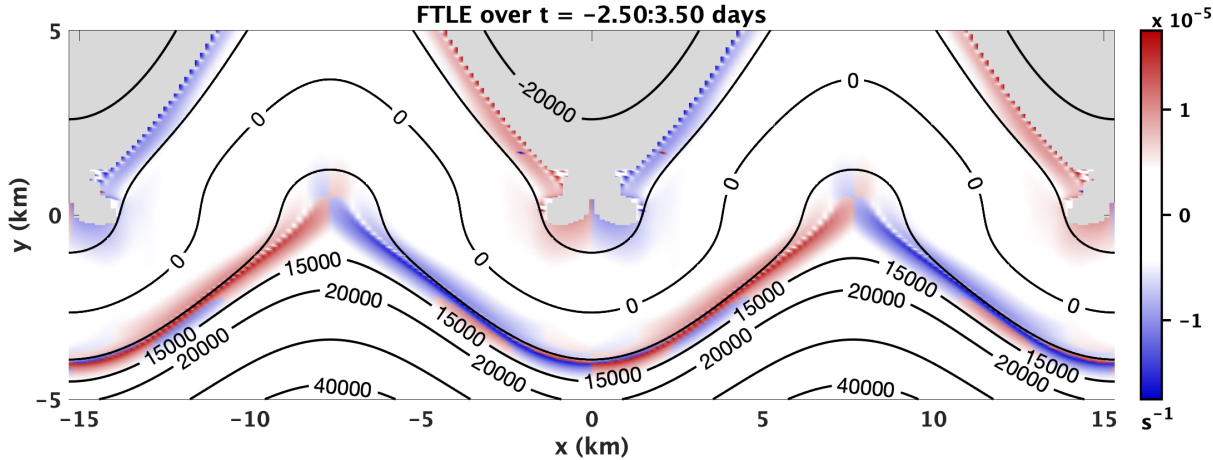

(c)

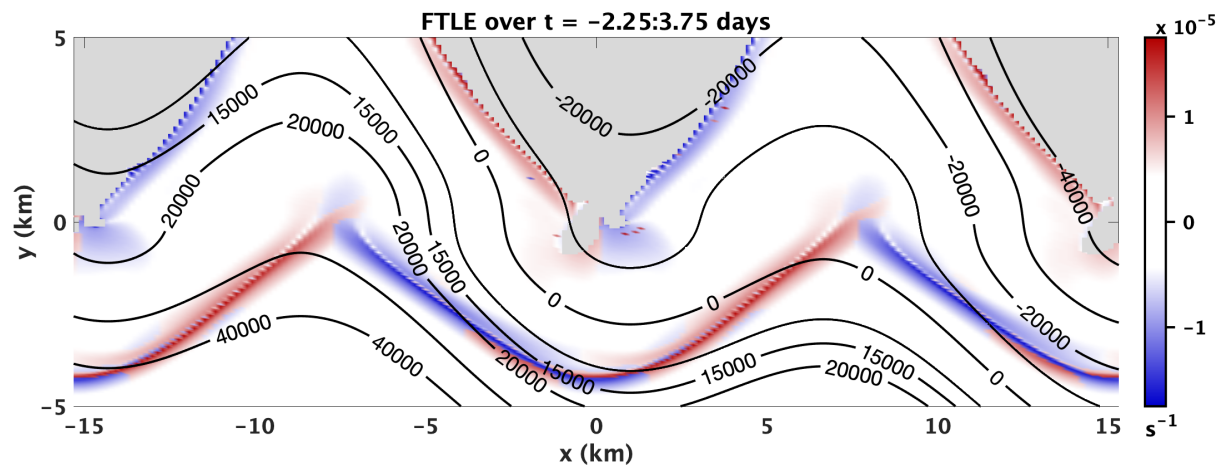

(d)

Figure 3. Finite-time Lyapunov exponent (FTLE) field for the base case parameters in Table 1, evaluated over the time interval from three days forward and backward, centered at (a) $t=0$, (b) $t=0.25$ days, (c) $t=0.5$ days, and (d) $t=0.75$ days. Backward-time FTLEs are shown at the blue end of the colormap, while forward-time FTLEs are displayed in red. Black contours show the volume-transport streamfunction at the center time. Grey shading indicates a lack of data due to boundary effects. 


\subsection{Transport Properties}

As described in Section 2.3, transport quantities are reported here relative to the $\Psi=0$ surface. The analysis time period is chosen to be identical to the wave period, or three tidal periods. It is generally not true that the net transport after two tidal periods is twice that after one tidal period. Therefore, we have not normalized the calculations by the time interval, which would be misleading in this regard.

Figure 4 shows the water masses that have been exchanged between on-shore and off-shore waters over one wave period. Figure $4 \mathrm{a}$, b show initial and final positions, respectively, of particles for a three day integration, colored by their initial distance from the coast. Figure $4 \mathrm{c}$ illustrates the evolution of the $\Psi=0$ boundary curve, with red areas indicating off-shore and cyan on-shore transport. Much of the observed mixing between shallower and deeper waters (Figure 4b) evidently occurs without crossing the $\Psi$ isoline. While this type of transport-across $y=0-$ may be of greater relevance for practical applications, such as coastline protection from pollutants, the results here are focused on the smaller but not insignificant transport across the Eulerian flow structure.

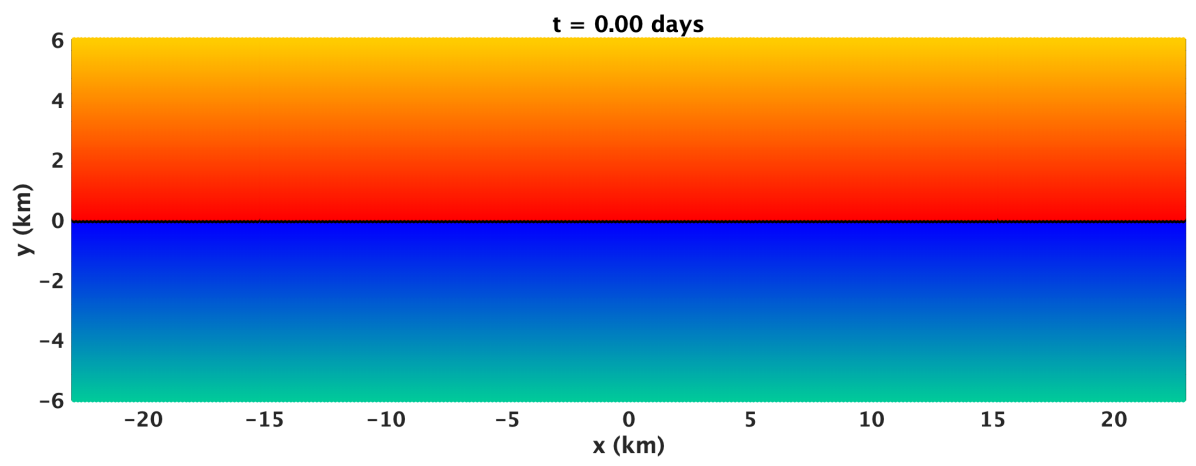

(a)

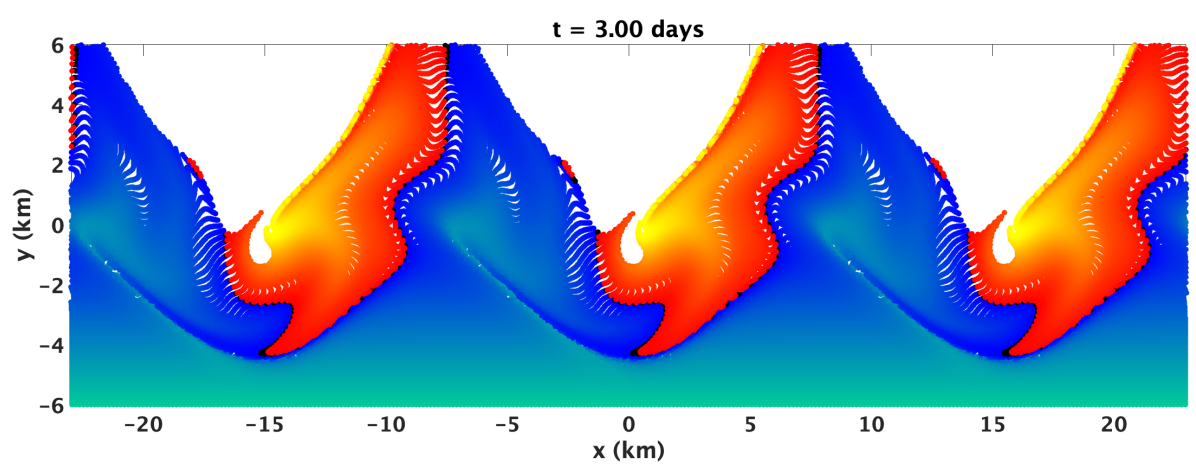

(b)

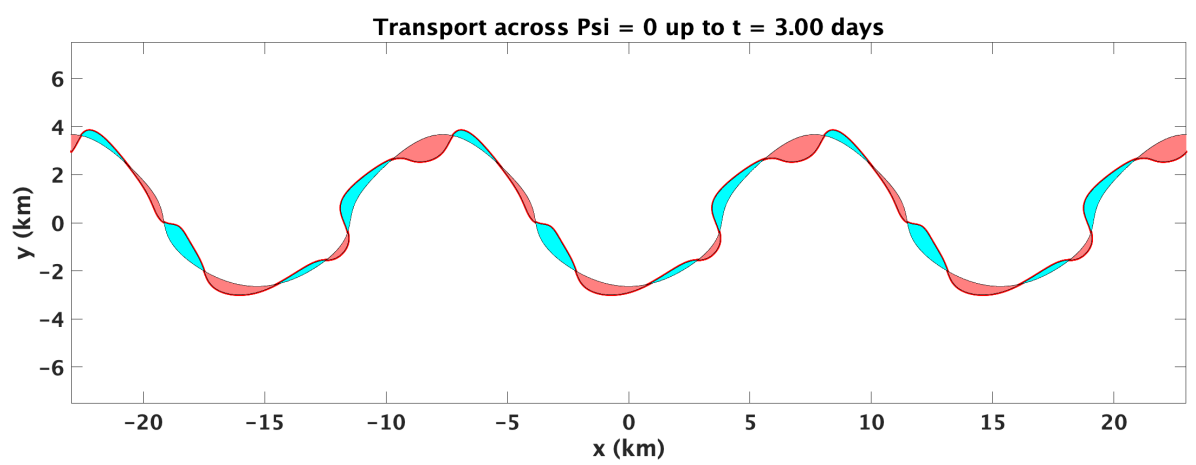

(c)

Figure 4. (a) Initial positions and (b) positions after three days of a set of sample trajectories, colored by their initial $y$-position. (c) Water masses transported off-shore (cyan) and on-shore (red) across $\Psi=0$ over the analysis period of three days. 
The patterns of the latter consist, in the base case, of four distinct lobes on each side of $\Psi=0$ in each interval of one wavelength. Over one wavelength (about $15.3 \mathrm{~km}$ ), the total transport amounts to $0.30 \mathrm{~km}^{3}$ in each direction. To put this into context, the total volume of water on the shelf $(\Psi<0)$ over one wavelength is $7.6 \mathrm{~km}^{3}$. Thus, the exchange constitutes a substantial portion, approximately $4 \%$. Even after one wave period, some filamentation of the exchanged water masses can be observed.

\section{Parameter Space Exploration}

The transport volume cited in Section 3.2 is specific to the model configuration for the base case defined by the parameters in Table 1. This raises the question of how the transport varies with the parameters. The ten-dimensional parameter space is too vast for exhaustive exploration. Therefore, we will instead focus on three secondary non-dimensional parameters that capture the relative importance of the different flow components. These are summarized in Table 2. Elements that are kept constant are the latitude $\phi$, the water depth at the peak of the coastal jet $h_{0}$, the tidal parameters $V_{\text {tide }}$ and $\omega$, and the analysis time interval $\tau$.

Table 2. Non-dimensional parameters, their definition, physical meaning, and base case values.

\begin{tabular}{llll}
\hline$\alpha$ & $\frac{c_{p}}{U}=\frac{2 \pi /(T k)}{U}$ & ratio of topographic wave speed to jet strength & -1.18 \\
$\delta$ & $\frac{\left(V / 2+V_{\text {tide }} / \pi\right) / \omega}{L}$ & ratio of cross-shelf velocity advective length scale to jet width & 1.63 \\
$\gamma$ & $\frac{h_{0} / S}{L}$ & ratio of shelf width to jet width & 2.0 \\
\hline
\end{tabular}

The non-dimensional parameters consider the topographic wave speed relative to the strength of the coastal jet $(\alpha)$, a cross-shelf velocity advective length scale (the integral of Equation (12) at $x=\pi / k$ and $y=0$ over half a tidal wave period) relative to the tidal amplitude $(\delta)$, and the shelf width relative to the coastal jet width $(\gamma)$. We chose these particular combinations of model parameters because they are based on easily measured quantities and because they reflect the competition of the relevant physical processes. In particular, $\alpha$ accounts for the relative strength of the two components of along-shore flow (see Equation (11)). The second non-dimensional parameter, $\delta$, considers the length scale the material has to move beyond the jet to be effectively mixed with coastal waters. Lastly, $\gamma$ accounts for the effectiveness of the shelf width to the jet width in cross-shelf transport.

Each of the non-dimensional parameters is varied over a range centered on the base case, capturing realistic values for the underlying physical variables. Transport volumes are computed for each case over three tidal periods and one wavelength interval by varying the numerator or denominator of each ratio. We have chosen to fix $\tau$, rather than varying it with $T$, for better comparability. Note that the wavelength $\Lambda$ is a function of $k$, which in turn varies with $S$ and $T$ (Equation (13)):

$$
\Lambda=\frac{2 \pi}{|k|}=\frac{4 \pi^{2} h_{0}}{S f T}
$$

Thus, the wavelength changes with $\alpha$ and $\gamma$ if $T$ and $S$ are varied, respectively.

Of course, not all ten physical parameters are captured by the three non-dimensional ones investigated, and so, the general parameter space is not completely explored here. Indeed, for each series, we chose to change the smallest number of physical parameters. One consequence of this is that $\alpha, \delta$, and $\gamma$ are not varied completely independently. In particular, $\gamma$ is linked to both of the other parameters: when $\gamma$ is varied by adjusting the numerator $(S)$ while keeping the denominator $(L)$ constant, it impacts $\alpha$, because $k$ and hence $c_{p}$ are a function of $S$. On the other hand, when $\gamma$ is varied by adjusting the denominator $(L)$, this changes $\delta$, which is directly a function of $L$. Varying $\delta$ by adjusting $L$, of course, has a similar effect on $\gamma$. However, $\alpha$ can be varied without impacting $\gamma$ by varying $T$ when adjusting the numerator. 
The results of the parameter space exploration are summarized in Figures 5-7, which also show how the corresponding physical parameters are varied for each experiment series. For those cases with near-linear dependence over the parameter space considered, linear fits are provided to quantify the strength of the parameter dependence. Figure 5 shows the two experiment series for $\alpha$, which measures the wave speed relative to the jet strength, varying the numerator and denominator separately. They nearly coincide, with a stronger correspondence than for the other two parameters. Apparently, this is due to the co-dependency on the other non-dimensional parameters mentioned above. This confirms that the ratio of the wave speed to jet strength is indeed a controlling parameter for the cross-shelf transport. In the parameter range explored here, the wave and jet act in opposite directions, and hence, $\alpha<0$. As their respective magnitudes approach each other $(\alpha=1)$, the strength of the combined along-shore flow acting as a barrier to cross-shelf transport weakens, allowing increased transport.
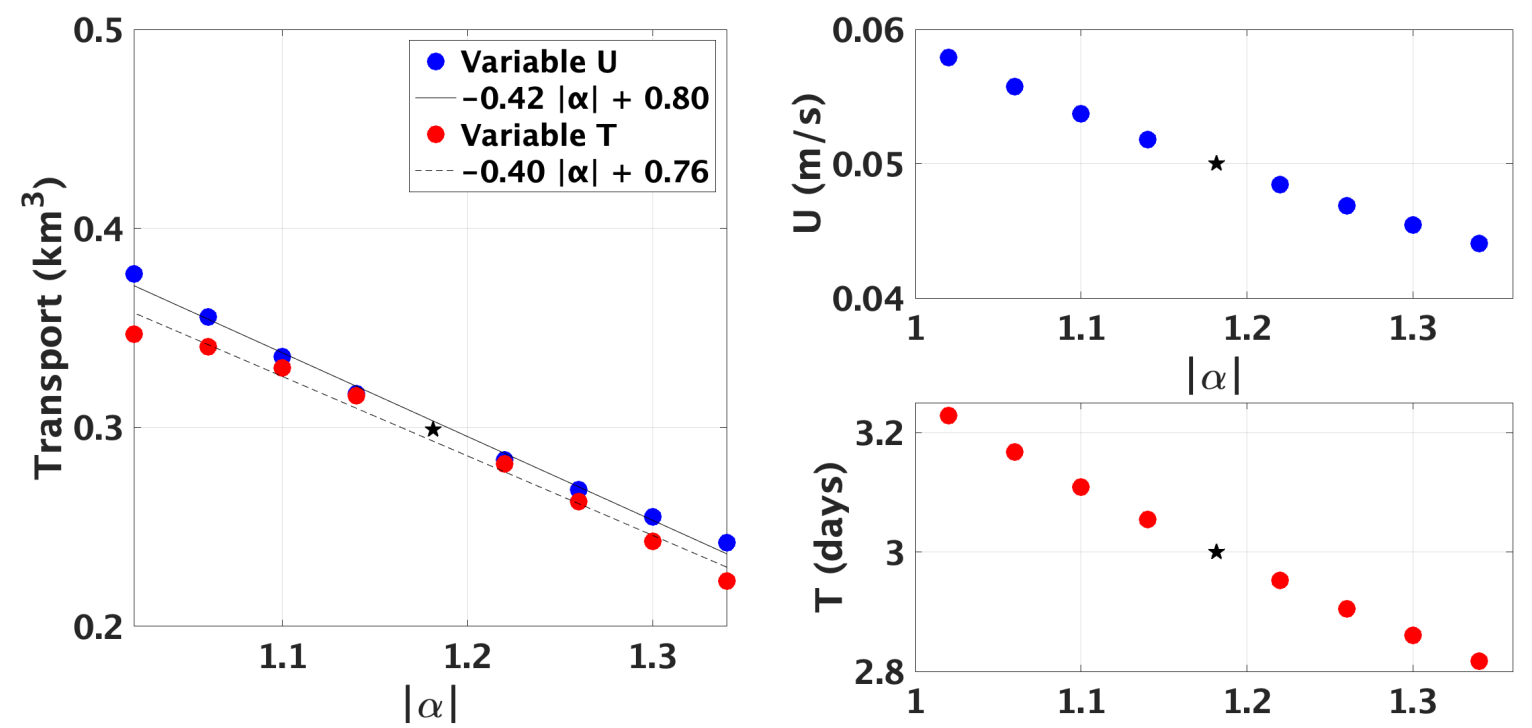

Figure 5. (Left) Cross-shelf transport across the surface $\Psi=0$ for a variety of values of $\alpha$, as defined in Table 2. The blue data points correspond to changes in the denominator and red data points to changes in the numerator. Lines show linear fits to the data series separately. (Right, top) Values of $U$ corresponding to the values of $|\alpha|$ used to derive the blue data points. (Right, bottom) Values of $T$ corresponding to the values of $|\alpha|$ used to derive the red data points. The black star in all panels shows the data point for the base case.

The dependency of the transport on $\delta$ is more subtle (Figure 6). $\delta$ measures the strength of the cross-shelf advection versus the width of the jet. It is observed that there is a sweet spot maximizing transport near $\delta=1.5$. The transport results from particles crossing the velocity gradients at the jet edge. Generally, for small $\delta$, as the jet is very wide or the cross-shelf advection weak, this process is inhibited. On the other hand, for very large $\delta$ as the jet becomes very narrow relative to the cross-shelf advection, much of the cross-shelf advection occurs outside the influence of the jet, leading to only periodic transport. It takes both along- and cross-shelf advective components to generate advective mixing, both along and across topography in this case. For $\delta$ at either extreme, only one component dominates. 

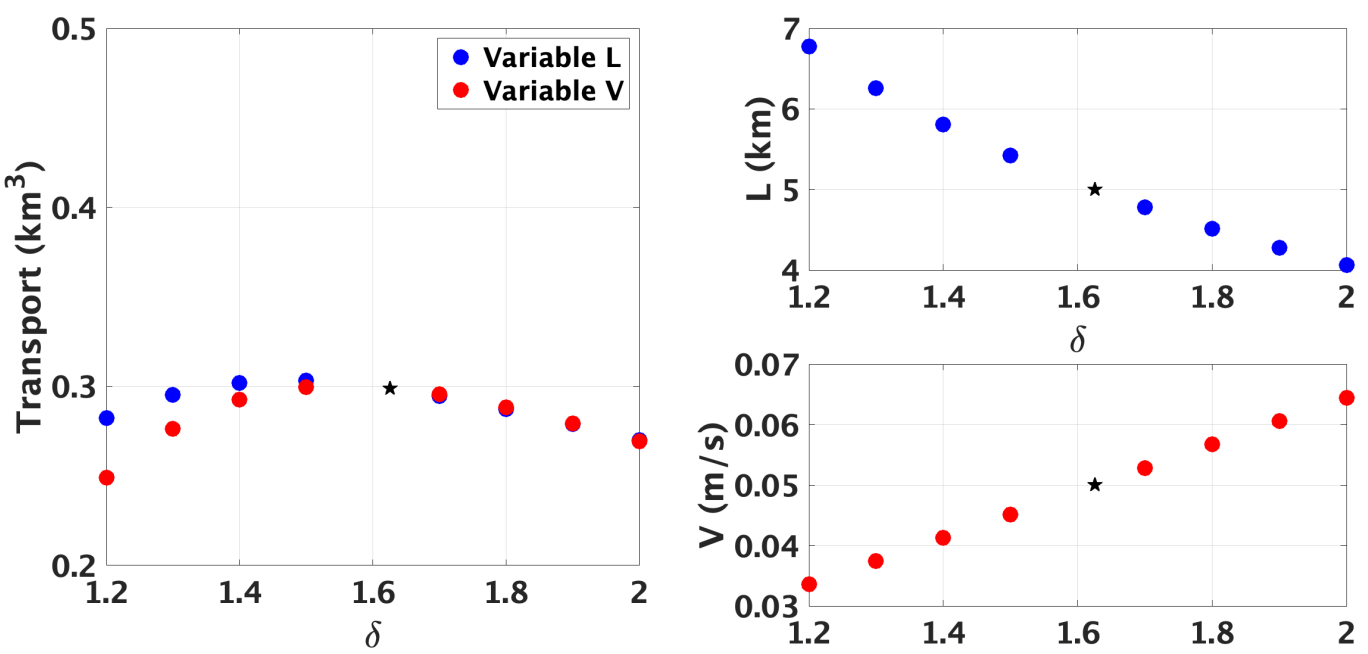

Figure 6. (Left) Cross-shelf transport across the surface $\Psi=0$ for a variety of values of $\delta$, as defined in Table 2. The blue data points correspond to changes in the denominator and red data points to changes in the numerator. (Right, top) Values of $L$ corresponding to the values of $\delta$ used to derive the blue data points. (Right, bottom) Values of $V$ corresponding to the values of $\delta$ used to derive the red data points. The black star in all panels shows the data point for the base case.

The trends of transport with $\gamma$ are considered in Figure 7. $\gamma$ measures the influence of the shelf width relative to the influence of the jet width. To make sense of these results, recall that an increasing $\gamma$ is accompanied by an increasing $\delta$ if $L$ is varied and by an increasing $|\alpha|$ if $S$ is varied (via changes in $c_{p}$; Equation (6)). It appears that for this part of the parameter space, these secondary effects dominate. For the case of constant $S$ and varying $L$, transport is fairly insensitive to $\gamma$, but a slight peak occurs around $\gamma=1.8$, where $\delta \approx 1.5$. In contrast, for constant $L$ and varying $S$, transport decreases with increasing $\gamma$ and correspondingly increasing $|\alpha|$, which leads to a strengthened barrier in the form of along-shore currents. Note that all the trends considered here are consistent with the fundamental idea that advective transport relies on a balance between cross-topography and along-topography advection.
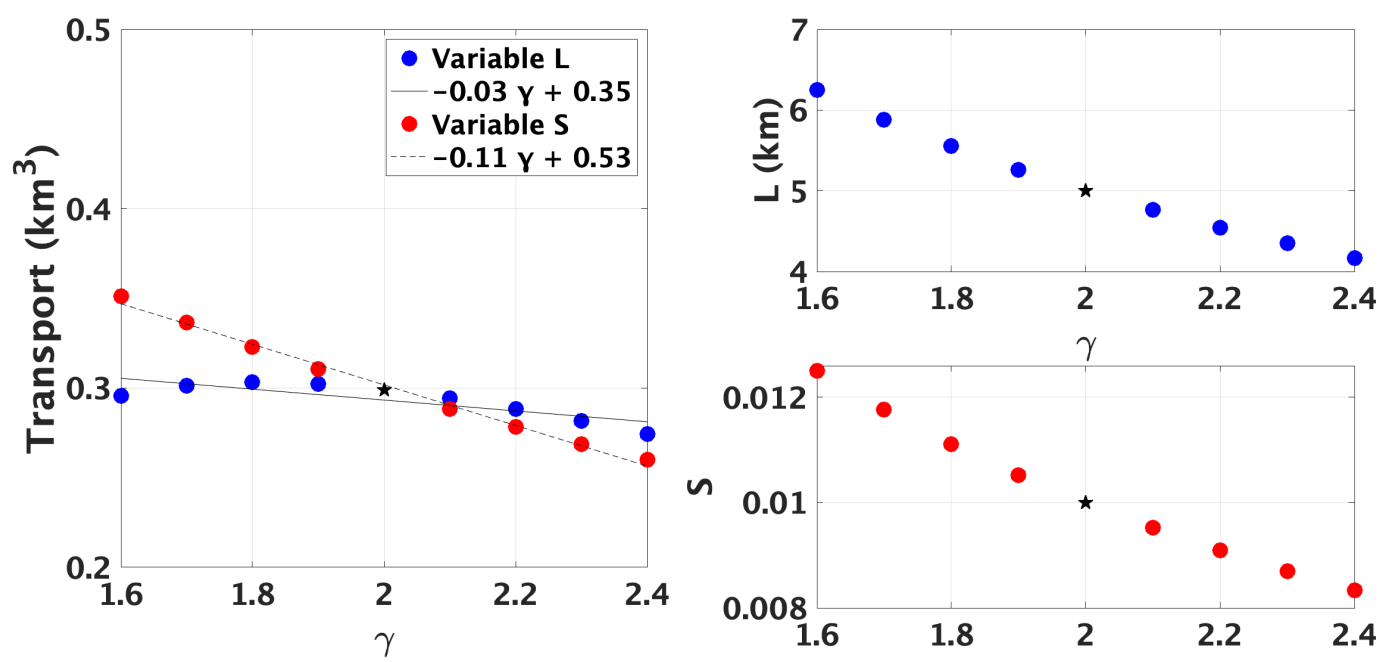

Figure 7. (Left) Cross-shelf transport across the surface $\Psi=0$ for a variety of values of $\gamma$, as defined in Table 2. The blue data points correspond to changes in the denominator and red data points to changes in the numerator. Lines show linear fits to the data series separately. (Right, top) Values of $L$ corresponding to the values of $\gamma$ used to derive the blue data points. (Right, bottom) Values of $S$ corresponding to the values of $\gamma$ used to derive the red data points. The black star in all panels shows the data point for the base case. 
Lastly, throughout this section, $V_{\text {tide }}$ has been kept constant. However, tidal currents can vary greatly between geographic locations. Thus, we independently explored the impact of varying $V_{\text {tide }}$ on the total transport. Predictably, stronger tidal currents were found to result in larger transport. In the range $V_{\text {tide }} \in[0.014,0.033] \mathrm{m} / \mathrm{s}$, transport increased from 0.22 to $0.46 \mathrm{~km}^{3}$, almost linearly with slope $12.7 \mathrm{~km}^{3} /(\mathrm{m} / \mathrm{s}$ ) (not shown).

\section{Application to the Texas Shelf}

To test the conclusion of significant cross-shelf transport due to the three processes captured in this transport model for a specific case with corresponding observed parameter values, we consider an example of flow along the Texas Shelf. In general, circulation on the Texas Shelf exhibits a strong wind-driven seasonal pattern [27-29]. During the winter months (October-March), a high pressure system typically sets up over Texas, inducing a down-coast wind field (blowing to the southwest, from Houston to Port Aransas). This wind field generates a down-coast oceanic circulation pattern, in which advection of buoyant river discharge and Ekman dynamics combine to create a strong $(0.5-0.75 \mathrm{~cm} / \mathrm{s})$ down-coast Texas Coastal Current. The downwelling signature of this circulation pattern is often masked by the homogenization of the water column due to strong wind induced mixing. Winter months are also characterized by the passage of strong fronts, which enhance such mixing and cause short-lived coastal current reversals (1-2 days) with higher variability closer to the Texas-Mexico border $\left(26^{\circ} \mathrm{N}\right)$. In contrast, the summer months (June-August) are characterized by up-coast wind patterns (wind blowing to the northeast, from Port Aransas to Houston). This up-coast wind pattern drives a "reversal" of the Texas Coastal Current, causing up-coast flow near the Texas coast. Due to the reduced strength of summer winds and the passage of fewer fronts, an upwelling circulation pattern can be observed. The transitional months September-October (up-coast to down-coast) and April-May (down-coast to up-coast) are characterized by high variability due to instabilities associated with flow transitions.

In addition to wind-induced circulation, the Texas coast is also influenced by river discharge [30] and offshore eddies impinging on the coastal zone [31-33]. Freshwater plumes can act as barriers to cross-shelf transport [34]. High river output has also been linked to increased offshore transport [30]. Offshore eddies in this area are typically between 100 and $300 \mathrm{~km}$ in diameter, extend up to $1000 \mathrm{~m}$ in depth, have circulation velocities of a few $\mathrm{cm} / \mathrm{s}$, and primarily influence the Texas coastal zone in two ways: direct impingement on the Texas Shelf or impingement on the Louisiana slope. If impinging directly on the Texas Shelf, the eddies are capable of overwhelming the ambient circulation pattern and creating significant cross-topography transport. If impinging on the Louisiana slope, they can generate topographically controlled slope currents and topographic waves, which travel along the slope towards Texas [35-41].

Here, we consider a unique dataset of bottom current measurements spanning late-May to mid-June 2016. This cross-topography array of SeaHorse tilt current meters (TCMs) was deployed between the $30 \mathrm{~m}$ and $200 \mathrm{~m}$ isobaths, although most instruments were concentrated in the $50-100 \mathrm{~m}$ depth range (Figure 8a). Despite the relatively short duration of this deployment, the dataset captured flow that shares several key aspects with our transport model: an approximately $20 \mathrm{~km}$ wide, topographically controlled jet with peak velocity of approximately $7 \mathrm{~cm} / \mathrm{s}$ is observed to be centered near the $50 \mathrm{~m}$ isobath. The jet is perturbed by a strong seven day oscillation (Figure $8 \mathrm{~b}$ ) with an approximate amplitude of $10 \mathrm{~cm} / \mathrm{s}$, consistent with a bottom-intensified topographic Rossby wave (the spectrum in Figure $8 \mathrm{~b}$ was calculated for instrument M7 at $70 \mathrm{~m}$ depth but is representative of all observed spectra). The jet is also perturbed by a weaker (approximately $5 \mathrm{~cm} / \mathrm{s}$ ) higher frequency oscillation with a period of one day, consistent with a tidal signal (Figure 8b). Note that, contrary to typical topographically controlled flows, which have shallow water to the right of the jet, here, the jet is reversed, with shallow water to the left, as is normal for the summertime Texas Shelf circulation. Furthermore, these observations are bottom current measurements taken $1 \mathrm{~m}$ off the bottom. Thus, the observed shoreward slant of the mean velocity vectors is possibly an Ekman spiral effect. 


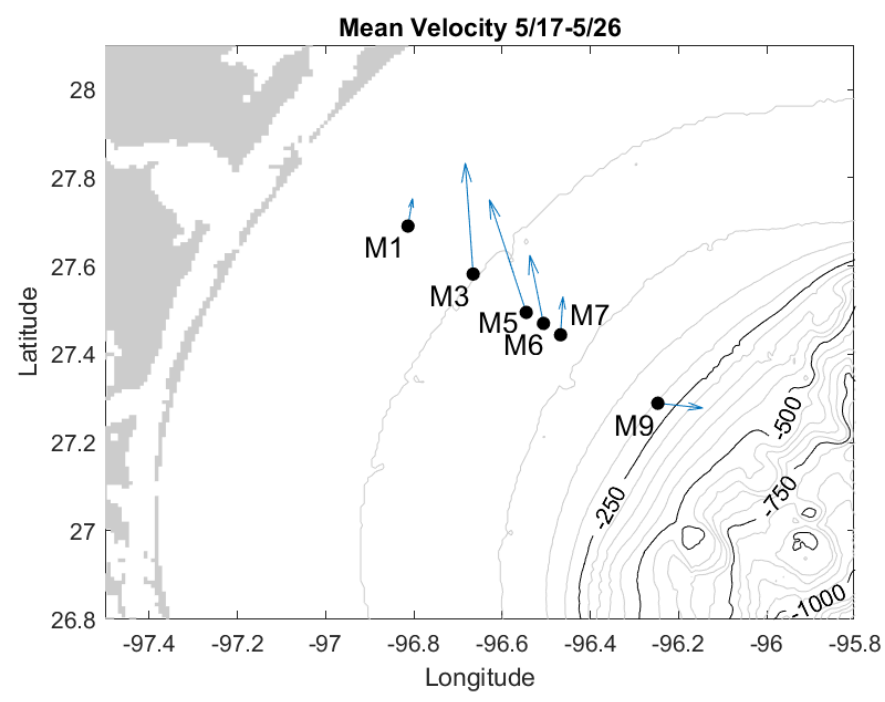

(a)

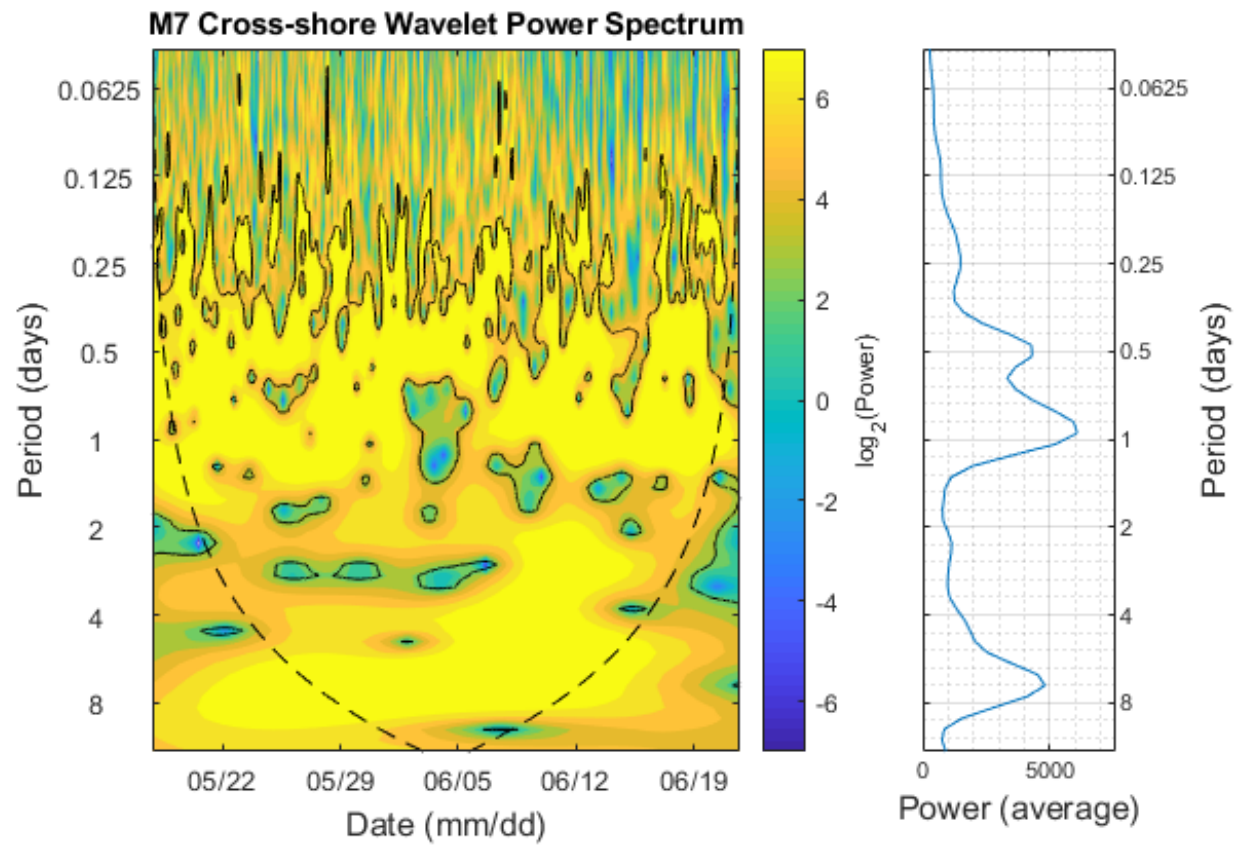

(b)

Figure 8. (a) Locations of the bottom current meters off the Texas coast. Grey lines show isobaths at $50 \mathrm{~m}$ intervals, with darker lines at $250 \mathrm{~m}$ intervals. Blue arrows indicate the mean flow. (b) Wavelet analysis at location M7 of the cross-shore current component with (left) the power spectrum as a function of time and (right) the global power spectrum. The solid black contour indicates where power becomes significant. The dashed line indicates the cone of influence, where anything "below" is potentially affected by edge effects.

To model this particular flow, we use the following parameter values: $\phi=27.5^{\circ}, h_{0}=50 \mathrm{~m}$, $S=0.0012, U=-0.07 \mathrm{~m} / \mathrm{s}, L=20 \mathrm{~km}, V=0.1 \mathrm{~m} / \mathrm{s}, T=7$ days, $V_{\text {tide }}=0.05 \mathrm{~m} / \mathrm{s}$, and $\omega=1$ day $^{-1}$. After one tidal period, the transport across $\Psi=0$ consists of a per wavelength $(\Lambda=40.39 \mathrm{~km})$ volume exchange of $0.38 \mathrm{~km}^{3}$ (Figure $9 \mathrm{a}$ ). The transport occurs in small pockets along the edge of the $\Psi=0$ surface. Over one wave period (or seven tidal cycles), the transport increases, with a per wavelength volume exchange of $0.46 \mathrm{~km}^{3}$ (Figure $9 \mathrm{~b}$ ). This suggests there is potential for significant 
background cross-topography transport on the Texas Shelf under normal conditions even without eddy impingement.

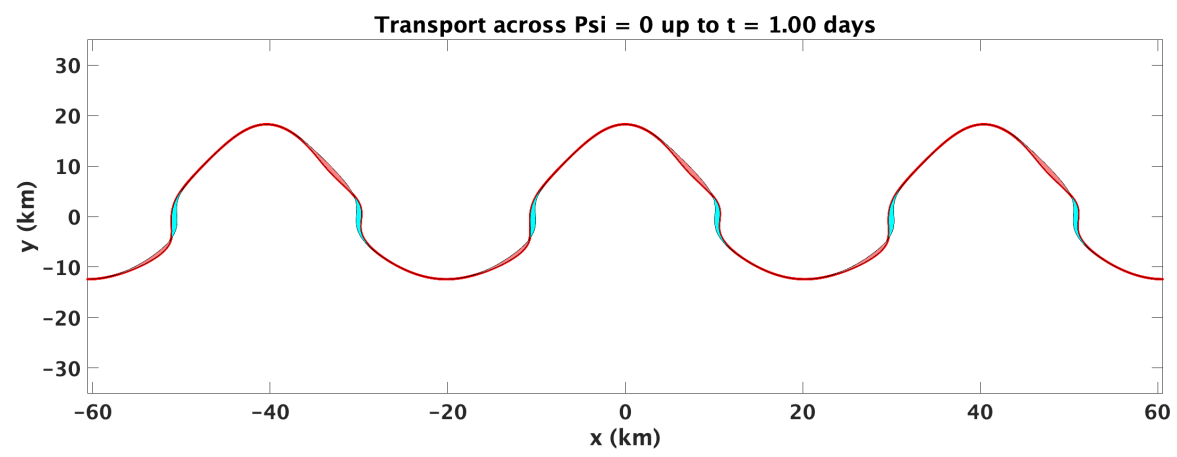

(a)

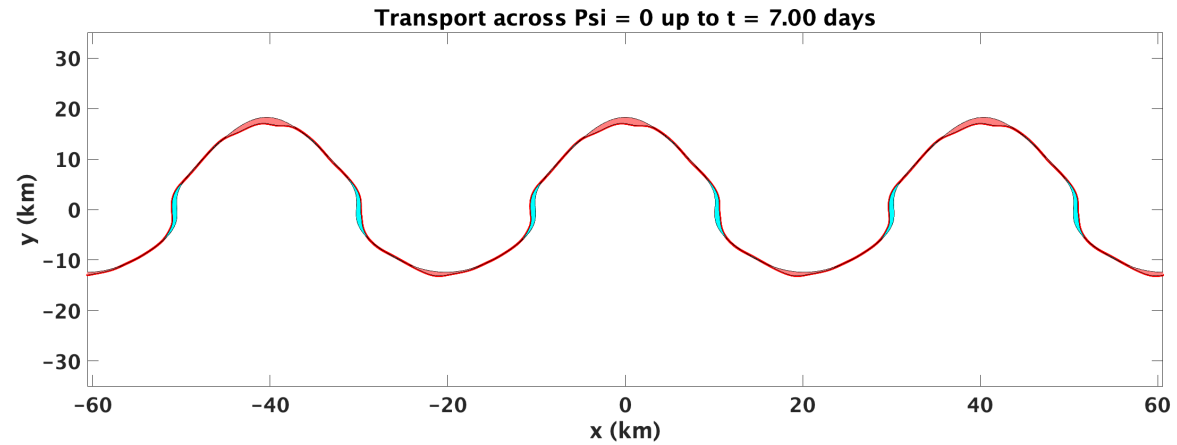

(b)

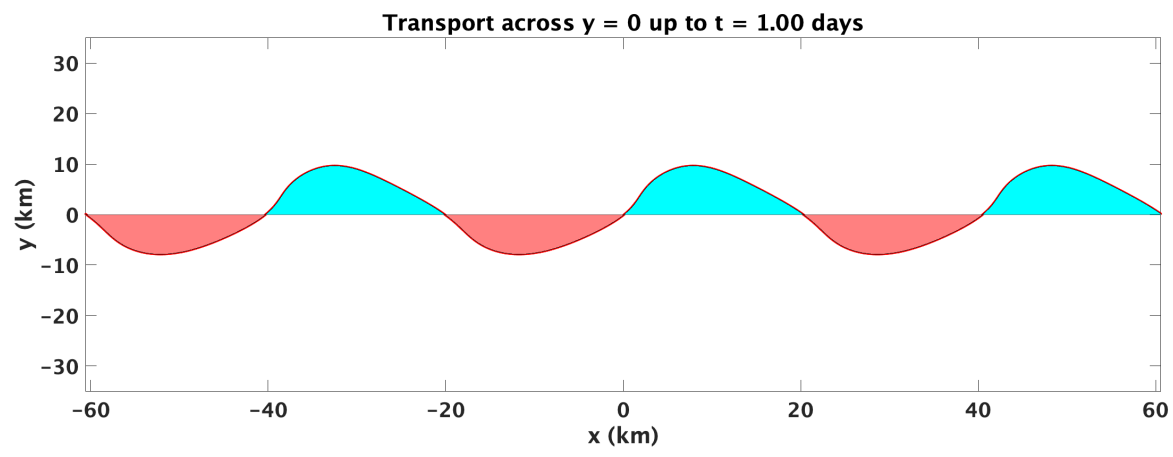

(c)

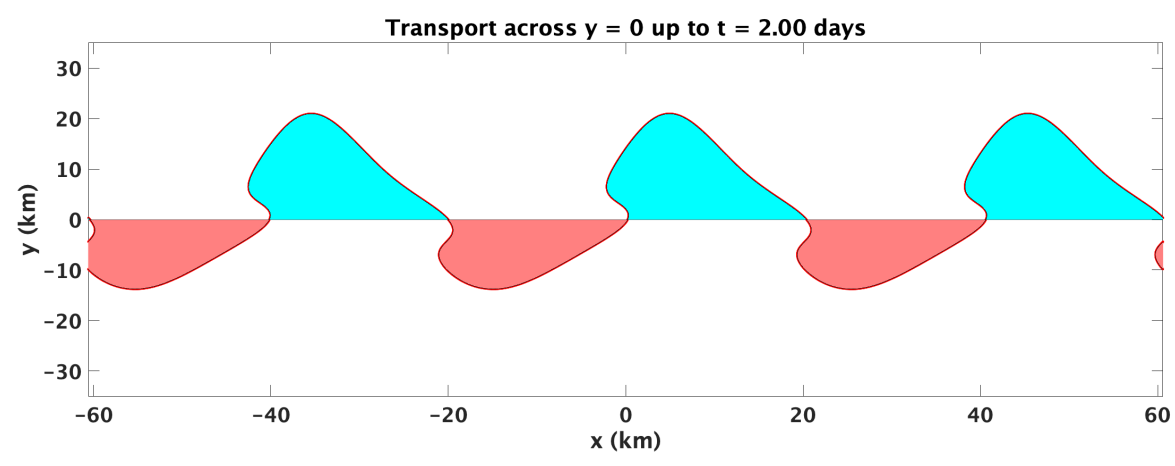

(d)

Figure 9. Cross-shelf transport for the case matching observed parameters for (a) one tidal period across $\Psi=0$, (b) one wave period (seven tidal periods) across $\Psi=0$, (c) one tidal period across $y=0$, and (d) two tidal periods across $y=0$. Red indicates water masses transported on-shore and cyan those moved off-shore. 
We return now to the earlier observation that for practical applications, the transport across a fixed geographic boundary, such as an isobath, may be more relevant. Figure $9 \mathrm{c}, \mathrm{d}$ show the transport over $y=0$ (the $50 \mathrm{~m}$ isobath) for one and two tidal periods, respectively. By this metric, onshore transport is even more substantial, at $5.55 \mathrm{~km}^{3}$ after one day and $11.11 \mathrm{~km}^{3}$ after two days.

\section{Discussion and Conclusions}

We presented a simple analytical model to analyze cross-shelf transport by generic shelf/slope dynamical processes. It consists of a linear superposition of a topographically controlled jet, a topographic Rossby wave, and a tidal oscillation. Despite its simplicity, the resulting transport characteristics are complex. Most importantly, the analysis demonstrates the potential for significant background cross-topographic transport under what are generally considered "normal" periodic shelf/slope conditions, without requiring the introduction of eddy impingement, frontal instabilities, or atmospheric forcing. In fact, the transport magnitude estimated here is comparable to that found in previous studies to result from eddy-shelf interactions [1].

To further probe these transport characteristics, we explored the parametric dependence of the transport on several key system parameters $\alpha, \delta$, and $\gamma$, whose definitions are designed to capture the main processes at work while relying on easily observable quantities. Due to the number of parameters involved in our model, the non-dimensional parameters chosen are not completely independent, but do illustrate how the trends in transport depend on these common ocean observables.

Each flow component independently will not result in any aperiodic cross-topography transport. The jet is directed along isobaths, and each individual oscillation is periodic, so particle trajectories will return to their initial position once every period. There is a slight drift due to the topographic slope, but that is not a significant factor. A combination of tidal oscillation and the Rossby wave will only produce reversible transport in a period that is a multiple of the two underlying periods. The combination of a tidal oscillation and a coastal jet will also result in particle trajectories that return to their initial depth contour (plus downstream advection) because the velocity components are orthogonal and the tidal signal is modeled as independent of location. It is the combination of the along-shore current with a spatially dependent cross-shore component that results in appreciable cross-topographic transport and consequent potential for mixing between coastal and open ocean water masses. Without the tidal component, however, that is in the case with only a jet and topographic wave, such transport across the $y=0$ line is somewhat artificial: in the moving reference frame, the streamfunction is constant as in Figure $2 \mathrm{~b}$, so that there is no cross-shelf transport across the $\Psi=0$ isoline. Thus, the combination of all three processes is necessary to produce true open coastal ocean mixing. As the tidal oscillation and stationary flow patterns are not orthogonal, there will be aperiodic transport. The effect is a time-dependent streamfunction field that is aligned with the relevant invariant manifolds only once per tidal cycle, as illustrated in Figure 3, leading to particles not returning to their initial positions after a tidal or wave period (Figure 4).

The transport volume is controlled by the interaction between the three processes with the topographic conditions. This is shown in the parameter sensitivity study. Parameter $\alpha$ considers the ratio of the topographic wave speed to the jet strength (Figure 5). As the wave speed and jet speed get closer to balancing each other, the transport increases. This occurs since the jet and wave speed counteract each other, and when neither dominates, the strength of the barrier die to along-shore currents is weakened. Parameter $\delta$ considers the ratio of the cross-shelf velocity advective length scale to the jet width (Figure 6). A sweet spot was identified in which the cross-stream and along-stream advective components couple to result in a maximum transport. Parameter $\gamma$ considers the ratio of the shelf width to the jet width (Figure 7). However, the secondary effects of accompanying changes in $\alpha$ and $\delta$ were found to dominate. The impact of increased tidal strength was tested in addition to the non-dimensional parameters and found to be associated with increased transport.

As a specific example, a transport analysis for the Texas coast is presented, using parameters drawn from observational data. The key features of this observational dataset are a topographically 
controlled jet, a one day oscillation consistent with a tidal signal, and a seven day oscillation, consistent with a topographic Rossby wave. The application of our model to these observational conditions suggests that a significant amount of cross-topographic transport (open-ocean coastal-ocean mixing) can occur under normal shelf conditions. This has implications for larval distribution, oil spill response, and other coastal management issues, as the absence of eddies or density filaments will not guarantee isolation of on-shore and off-shore waters.

In summary, it was shown that there exists a fundamental background open ocean coastal-ocean connectivity pathway due to the interactions of a topographically controlled jet, a topographic Rossby wave, and a tidal oscillation. An analytic model of such systems was provided, and a parameter study was conducted to explore the sensitivity of transport to several key ocean observables. The application of the model to the Texas Shelf demonstrated significant transport and mixing potential under these basic flow conditions. The parameter space exploration suggests that this finding will vary across other regions of the ocean, but it also enables straightforward transport predictions based on a few observable characteristics. It is the authors' hope that confirmation of this advective open-ocean coastal-ocean transport pathway will help inform coastal ocean management decisions and provide guidance for future field programs targeting coastal transport processes.

Author Contributions: Conceptualization, J.J.K., H.S.H., and A.D.K.; methodology, J.J.K.; software, H.S.H.; formal analysis and investigation, H.S.H., C.W.M., J.J.K., and A.D.K.; data curation, J.K and C.W.M.; writing, original draft preparation, review, and editing, H.S.H., J.J.K., and A.D.K.; funding acquisition, H.S.H., J.J.K., and A.D.K. All authors read and agreed to the submitted version of the manuscript.

Funding: This research was supported in part by a grant from the Gulf of Mexico Research Initiative to the Consortium for Advanced Research on the Transport of Hydrocarbons in the Environment (CARTHE), by the Texas General Land Office, Oil Spill Program (Program Manager: S. Buschang) under TGLO Contract Numbers 16-019-0009283 and 18-130-000-A670, by the Office of Naval Research under the CALYPSO DRI by Grant N00014-18-1-2461, and by the National Academies of Science, Engineering and Medicine's Gulf Research Program under Grants 2000009918 and 200011071.

Acknowledgments: The authors wish to thank two anonymous reviewers, whose insightful comments led to an improved manuscript. Model data can be replicated by solving the provided equations. Observational data are publicly available through the Gulf of Mexico Research Initiative Information \& Data Cooperative (GRIIDC) at https: / / data.gulfresearchinitiative.org under DOI 10.7266/9VP7F5KM.

Conflicts of Interest: The authors declare no conflict of interest. The funders had no role in the design of the study; in the collection, analyses, or interpretation of data; in the writing of the manuscript; nor in the decision to publish the results.

\section{References}

1. Brink, K.H. Cross-Shelf Exchange. Annu. Rev. Mar. Sci. 2016, 8, 59-78. [CrossRef] [PubMed]

2. Huthnance, J.M. Circulation, exchange and water masses at the ocean margin: The role of physical processes at the shelf edge. Prog. Oceanogr. 1995, 35, 353-431. [CrossRef]

3. Brink, K.H. Continental shelf baroclinic instability. Part I: Relaxation from upwelling or downwelling. J. Phys. Oceanogr. 2016, 46, 551-568. [CrossRef]

4. Etter, P.C.; Ulm, W.F.; Cochrane, J.D. The relationship of wind stress to heat flux divergence of Texas-Louisiana shelf waters. Cont. Shelf Res. 1985, 4, 547-552. [CrossRef]

5. Etter, P.C.; Howard, M.K.; Cochrane, J.D. Heat and freshwater budgets of the Texas-Louisiana shelf. J. Geophys. Res. Oceans 2004, 109, C02024. [CrossRef]

6. Weisberg, R.H.; Lianyuan, Z.; Liu, Y. On the movement of Deepwater Horizon Oil to northern Gulf beaches. Ocean Model. 2017, 111, 81-97. [CrossRef]

7. Bower, A.S. A Simple Kinematic Mechanism for Mixing Fluid Parcels Across a Meandering Jet. J. Phys. Oceanogr. 1991, 21, 173-180. [CrossRef]

8. Samelson, R.M. Fluid Exchange Across a Meandering Jet. J. Phys. Oceanogr. 1992, 22, 431-440. [CrossRef]

9. Rogerson, A.M.; Miller, P.D.; Pratt, L.J.; Jones, C.K.R.T. Lagrangian motion and fluid exchange in a barotropic meandering jet. J. Phys. Oceanogr. 1999, 29, 2635-2655. [CrossRef]

10. Wiggins, S. The dynamical systems approach to Lagrangian transport in oceanic flows. Ann. Rev. Fluid Mech. 2005, 37, 295-328. [CrossRef] 
11. Haller, G. Lagrangian coherent structures. Ann. Rev. Fluid Mech 2015, 47, 137-162. [CrossRef]

12. Samelson, R.M.; Wiggins, S. Lagrangian Transport in Geophysical Jets and Waves: The Dynamical Systems Approach; Springer Science \& Business Media: New York, NY, USA, 2006; Vlume 31. [CrossRef]

13. Budyansky, M.V.; Uleysky, M.Y.; Prants, S.V. Detection of barriers to cross-jet Lagrangian transport and its destruction in a meandering flow. Phys. Rev. E 2009, 79, 17. [CrossRef] [PubMed]

14. Pratt, L.; Barkan, R.; Rypina, I. Scalar flux kinematics. Fluids 2016, 1, 27. [CrossRef]

15. Kuehl, J.J. An analytic solution for barotropic flow along a variable slope topography. Geophys. Res. Lett. 2014, 41, 7591-7594. [CrossRef]

16. Ibanez, R.; Kuehl, J.J.; Shrestha, K.; Anderson, W. Brief communication: A nonlinear self-similar solution to barotropic flow over varying topography. Nonlinear Processes Geophys. 2018, 25, 201-205. [CrossRef]

17. Kuehl, J.J.; McMahon, C. An analytic solution for bottom intensified flow along sloping topography. Eur. J. Mech. B. Fluids 2020, 82, 156-160. [CrossRef]

18. Zavala Sansón, L.; van Heijst, G.J.F. Ekman effects in a rotating flow over bottom topography. J. Fluid Mech. 2002, 471, 239-255. [CrossRef]

19. Kuehl, J.J.; Sheremet, V.A. Two-layer gap-leaping oceanic boundary currents: Experimental investigation. J. Fluid Mech. 2014, 740, 97-113. [CrossRef]

20. Cushman-Roisin, B. Introduction to Geophysical Fluid Dynamics; Prentice-Hall: Englewood Cliffs, NJ, USA, 1994.

21. Shadden, S.C.; Lekien, F.; Marsden, J.E. Definition and properties of Lagrangian coherent structures from finite-time Lyapunov exponents in two-dimensional aperiodic flows. Physica D 2005, 212, 271-304. [CrossRef]

22. Branicki, M.; Wiggins, S. Finite-time Lagrangian transport analysis: Stable and unstable manifolds of hyperbolic trajectories and finite-time Lyapunov exponents. Nonlinear Processes Geophys. 2010, 17, 1-36. [CrossRef]

23. Huntley, H.S.; Lipphardt, B.L., Jr.; Jacobs, G.A.; Kirwan, A.D., Jr. Clusters, deformation, and dilation: Diagnostics for material accumulation regions. J. Geophys. Res. Oceans 2015, 120, 6622-6636. [CrossRef]

24. Coulliette, C.; Wiggins, S. Intergyre transport in a wind-driven, quasigeostrophic double gyre: An application of lobe dynamics. Nonlinear Processes Geophys. 2001, 8, 69-94. [CrossRef]

25. Mancho, A.M.; Hernández-García, E.; Small, D.; Wiggins, S.; Fernández, V. Lagrangian transport through an ocean front in the northwestern Mediterranean sea. J. Phys. Oceanogr. 2008, 38, 1222-1237. [CrossRef]

26. Brett, G.J.; Pratt, L.J.; Rypina, I.I.; Sánchez-Garrido, J. The Western Alboran Gyre: An analysis of its properties and its exchange with surrounding water. J. Phys. Oceanogr. 2020. [CrossRef]

27. Cochrane, J.D.; Kelly, F.J. Low-frequency circulation on the Texas-Louisiana continental shelf. J. Geophys. Res. 1986, 91, 10645-10659. [CrossRef]

28. Nowlin, W.D.; Jochens, A.E.; DiMarco, S.F.; Reid, R.O.; Howard, M.K. Low-Frequency Circulation Over the Texas-Louisiana Continental Shelf. In Circulation in the Gulf of Mexico: Observations and Models; Sturges, W., Lugo-Fernández, A., Eds.; American Geophysical Union: Washington, DC, USA, 2005; pp. 219-240. [CrossRef]

29. Hetland, R.D.; DiMarco, S.F. How does the character of oxygen demand control the structure of hypoxia on the Texas-Louisiana continental shelf? J. Mar. Syst. 2008, 70, 49-62. [CrossRef]

30. Thyng, K.M.; Hetland, R.D. Seasonal and interannual cross-shelf transport over the Texas and Louisiana continental shelf. Cont. Shelf Res. 2018, 160, 23-35. [CrossRef]

31. Forristall, G.Z.; Schaudt, K.J.; Cooper, C.K. Evolution and kinematics of a loop current eddy in the Gulf of Mexico during 1985. J. Geophys. Res. 1992, 97, 2173-2184. [CrossRef]

32. Schmitz, W., Jr. Cyclones and westward propagation in the shedding of anticyclonic rings from the Loop Current. In Circulation in the Gulf of Mexico: Observations and Models; Sturges, W., Lugo-Fernández, A., Eds.; American Geophysical Union: Washington, DC, USA, 2005; pp. 241-261. [CrossRef]

33. Biggs, D.C.; Jochens, A.E.; Howard, M.K.; DiMarco, S.F.; Mullin, K.D.; Leben, R.R.; Muller-Karger, F.E.; Hu, C. Eddy Forced Variations in On- and off-Margin Summertime Circulation Along the 1000-m Isobath of the Northern Gulf of Mexico, 2000-2003, and Links with Sperm Whale Distributions Along the Middle Slope. In Circulation in the Gulf of Mexico: Observations and Models; Sturges, W., Lugo-Fernández, A., Eds.; American Geophysical Union: Washington, DC, USA, 2005; pp. 71-85. [CrossRef]

34. Roth, M.K.; MacMahan, J.; Reniers, A.J.H.M.; Özgökmen, T.M.; Woodall, K.; Haus, B.K. Observations of inner shelf cross-shore surface material transport adjacent to a coastal inlet in the northern Gulf of Mexico. Cont. Shelf Res. 2017, 137, 142-153. [CrossRef] 
35. Walker, N. Wind and eddy-related shelf/slope processing and coastal upwelling. In Circulation in the Gulf of Mexico: Observations and Models; Sturges, W., Lugo-Fernández, A., Eds.; American Geophysical Union: Washington, DC, USA, 2005; pp. 295-313. [CrossRef]

36. Hetland, R.; Hsueh, Y.; Yuan, D. On the decay of a baroclinic jet flowing along a continental slope. J. Geophys. Res. Oceans 2001, 106, 19797-19807. [CrossRef]

37. Hamilton, P.; Fargion, G.S.; Biggs, D.C. Loop Curren Eddy Paths in the Western Gulf of Mexico. J. Phys. Oceanogr. 1999, 29, 1180-1207. [CrossRef]

38. Hamilton, P. Topographic Rossby waves in the Gulf of Mexico. Prog. Oceanogr. 2009, 82, 1-31. [CrossRef]

39. Hamilton, P. Deep-Current Variability near the Sigsbee Escarpment in the Gulf of Mexico. J. Phys. Oceanogr. 2007, 37, 708-726. [CrossRef]

40. Hamilton, P.; Lugo-Fernández, A. Observations of high speed deep currents in the northern Gulf of Mexico. Geophys. Res. Lett. 2001, 28, 2867-2870. [CrossRef]

41. Kuehl, J.; DiMarco, S.; Spencer, L.; Guinasso, N., Jr. Application of the smooth orthogonal decomposition to oceanographic data sets. Geophys. Res. Lett. 2014, 41, 3966-3971. [CrossRef]

Publisher's Note: MDPI stays neutral with regard to jurisdictional claims in published maps and institutional affiliations.

(C) 2020 by the authors. Licensee MDPI, Basel, Switzerland. This article is an open access article distributed under the terms and conditions of the Creative Commons Attribution (CC BY) license (http:/ / creativecommons.org/licenses/by/4.0/). 